Bond University

Research Repository

\title{
The role of fear avoidance beliefs in return to work post-injury
}

Watt, Bruce D.; Ford, Lucas A.; Doley, Rebekah M.; Ong, Sabrina; Fritzon, Katarina; Hicks, Richard E.; Cacciola, Tony

Published in:

Journal of Vocational Rehabilitation

DOI:

10.3233/JVR-150756

Licence:

Unspecified

Link to output in Bond University research repository.

Recommended citation(APA):

Watt, B. D., Ford, L. A., Doley, R. M., Ong, S., Fritzon, K., Hicks, R. E., \& Cacciola, T. (2015). The role of fear avoidance beliefs in return to work post-injury. Journal of Vocational Rehabilitation, 43(1), 75-82.

https://doi.org/10.3233/JVR-150756

\section{General rights}

Copyright and moral rights for the publications made accessible in the public portal are retained by the authors and/or other copyright owners and it is a condition of accessing publications that users recognise and abide by the legal requirements associated with these rights.

For more information, or if you believe that this document breaches copyright, please contact the Bond University research repository coordinator. 
The Role of Fear Avoidance Beliefs in Return to Work Post-Injury

Bruce D. Watt ${ }^{\mathrm{a}}$, Lucas A. Ford ${ }^{\mathrm{a}}$, Rebekah M. Doley ${ }^{\mathrm{a}}$, Sabrina Ong ${ }^{\mathrm{a}}$, Katarina Fritzon ${ }^{\mathrm{a}}$, Richard E. Hicks ${ }^{\mathrm{a}}$, and Tony Cacciola ${ }^{\mathrm{b}}$

${ }^{a}$ Bond University, Queensland, Australia

${ }^{\mathrm{b}}$ Wesfarmers

Correspondence to Bruce Watt, Faculty of Humanities and Social Sciences, Bond University, Gold Coast, QLD 4229 Australia, Ph 6175595 2653, Fax 61755952540 Email bwatt@bond.edu.au 


\begin{abstract}
BACKGROUND: Fear avoidance beliefs have been demonstrated significant predictors of disability and work status post occupational injury and appear particularly important in explaining the transition from acute to chronic disability. OBJECTIVE: This study examined the relationship between fear avoidance beliefs, health-related quality of life, and their influence on return to work (RTW) outcomes, including durable RTW, postoccupational injury. METHODS: A total of 1179 questionnaires were posted to clients previously receiving vocational rehabilitation services from the Return to Work Assist program in Queensland, Australia. Participants were asked to indicate their current RTW status in addition to completing questionnaires measuring fear avoidance beliefs and physical and mental aspects of health related quality of life. RESULTS: The statistical analyses included 104 participants. ANOVA indicated the RTW group reported significantly better physical health than the no RTW (NRTW) group. No significant differences were observed between groups on mental health. ANOVA also indicated that participants with RTW outcomes reported significantly lower fear avoidance beliefs than the NRTW group. However, when controlling for physical health, no fear avoidance group differences were observed. Significant correlations were observed between fear avoidance beliefs and measures of physical and mental health. CONCLUSIONS: The findings are suggestive of a reciprocal relationship between fear avoidance beliefs, physical, and mental health in which fear avoidance beliefs hinder recovery from physical injury, contributing to deterioration in mental health. These findings also suggest that collaborative practice between medical practitioners, psychologists, and physiotherapists is likely to be required to improve RTW outcomes for this complex client group. Identifying and addressing fear avoidance beliefs early in the treatment process may decrease the likelihood of long-term disability and work absence.
\end{abstract}


Keywords: durable return to work, fear avoidance beliefs, health related quality of life 
The Role of Fear Avoidance Beliefs in RTW Post-Injury

Paid employment is an important aspect of life and can have a major impact on an individual's self-image, sense of personal worth, and social status (Szymanski \&Hershenson, 1998). Individuals who are absent from work for a prolonged period (18 months or more) have a heightened risk of economic and social deprivation and encounter significant barriers in returning to work (Dekkers-Sanchez, Wind, Sluiter, \& Frings-Dresen, 2010). In Australia, lost productivity and compensation claims amount to billions of dollars each year due to physical and psychological work-related injuries (Safework Australia, 2012).

One means of reducing costs associated with occupational injury involves identifying individuals at risk of long-term disability. While the likelihood of recovery post-injury and RTW in the first three to six weeks is high, a minority of injured workers (5 to 10\%) require additional assistance (Waddell, Burton, \& Kendall, 2008). A number of studies have indicated that psychosocial variables are of greater prognostic value in predicting chronic disability than traditional biomedical variables, such as physical impairment (Burton, Tillotson, \& Main, 1995; Macfarlane et al., 1999). Moreover, functional impairment may continue even when the source of pain has been addressed, suggesting that factors other than physicality are important contributors to disability (Turk, 2002). Of the psychosocial factors examined in the literature, fear avoidance beliefs may be the most powerful psychosocial predictor of disability with particular relevance in predicting prolonged work absence (Waddell, Newton, Henderson, Somerville, \& Main, 1993).

Following injury, individuals with heightened fear avoidance beliefs develop painrelated fear, or pain catastrophising, resulting in the avoidance of movements believed to be harmful. Waddell et al. (1993) argue: "Fear of pain and what we do about pain may be more disabling than pain itself” (p. 164). To explain the transition from acute to chronic pain, 
Vlaeyen and Linton (2000) developed a cognitive-behavioural model of chronic low back pain, known as the fear avoidance model. This model is based on the premise that the interpretation of pain can lead to two different behavioural pathways. When pain is interpreted as non-threatening, the individual is likely to continue engaging in his/her daily activities, promoting functional recovery. Conversely, when pain is interpreted as threatening (i.e., pain catastrophising), these interpretations result in pain-related fear and safety seeking behaviours, such as avoidance. While avoidance behaviours provide negative reinforcement and reduce fear in the short-term, they prevent the disconfirmation of the individual's belief (e.g., that he/she cannot engage in usual behaviours due to intolerable pain). There begins a vicious cycle, leading to delayed recovery, ongoing disability, and work absence. Consistent with this postulation, fear avoidance beliefs have been demonstrated as predictive of physical aspects of health-related quality of life (Keely et al., 2008). Such beliefs may also negatively impact on other aspects of quality of life, including withdrawal from social activities and mental health symptomatology (e.g., depression).

Vlaeyen and Linton’s (2000) model has received additional support within the literature. In a prospective study including a sample of 78 participants with work-related lower back pain, Fritz, George, and Delitto (2001) found that fear avoidance beliefs did not initially explain a significant proportion of variance in disability when controlling for pain intensity and physical impairment. However, when measured four weeks later, fear avoidance beliefs were significant predictors of disability and work status, even when controlling for pain intensity, physical impairment, and type of treatment received. Fritz et al. concluded that fear avoidance beliefs were an important factor in explaining the transition from acute to chronic disability. Highlighting the strong association between fear avoidance beliefs and return to work, intervention studies have demonstrated that improvements in painrelated cognitions, particularly pain catastrophising, have been associated with improved 
return to work outcomes (Sullivan, Adams, Thibault, Corbiere, \& Stanish, 2006). In addition, unemployed individuals have demonstrated more fear avoidance beliefs than their employed counterparts (Pfingsten, Kroner-Herwig, Leibing, Kronshage, \& Hildebrand, 2000).

Pfingsten et al. compared employed and unemployed participants, no studies to date have explored the relationship between fear avoidance beliefs and durable return to work. The current study investigated this relationship by comparing fear avoidance beliefs among injured workers with three different RTW outcomes: RTW, non-durable RTW (e.g., not currently working but a previous temporary RTW), and NRTW.

While most studies investigating fear avoidance beliefs have focused on lower back pain, the fear avoidance model has been applicable to a range of chronic pain conditions (Rose, Klenerman, Atchison, \& Slade, 1992). The current study included a wide range of injuries, including those classified as upper extremity, lower extremity, musculoskeletal, and multiple physical injuries. The aim of the current study was three-fold: (1) to compare physical and mental aspects of health-related quality of life according to RTW outcome in a sample where the majority of participants experienced prolonged work absence, (2) to further explore the relationship between fear avoidance beliefs and physical and mental aspects of health-related quality of life, and (3) to explore the relationship between fear avoidance beliefs and durable RTW. Given previous findings, it was hypothesised that the RTW group would report lower levels of fear avoidance beliefs and higher levels of health-related quality of life than participants with non-durable RTW and NRTW outcomes. It was also hypothesised that fear avoidance beliefs would be significantly related to physical and mental aspects of health-related quality of life. 


\section{Method}

\section{Study Location and Participants}

Participants were previous clients of the Return to Work Assist (RTWA) program operated by Q-Comp, an independent statutory authority established under the Workers' Compensation and Rehabilitation Act 2003 in Queensland, Australia. Q-Comp established RTWA to provide individualised support to workers with injuries which preclude them from returning to their previous employment. Injured workers can access RTWA services after their worker’s compensation claim has ceased. Given these circumstances, clients of RTWA have often experienced long-term unemployment following their workplace injury along with a number of barriers to successful RTW. RTWA also provides RTW services to injured workers with open claims for compensation against their employer. Under Section 267 of the Workers' Compensation and Rehabilitation Act 2003, injured workers with an open common law claim for compensation against their employer are mandated to satisfactorily participate in the RTWA program to mitigate their loss.

RTWA utilises a two-pronged approach to help clients RTW after sustaining workrelated injuries. The first is to prepare clients for re-employment through activities such as resume writing, job search, and interview training. Secondly, the program has adopted a career development approach to harness the potential of clients for prospective career changes, as well as to enhance career advancement.

The current study was conducted in the context of an independent evaluation of the RTWA program by the authors. A total of 1179 previous RTWA clients were randomly selected from the Q-Comp database to be included in this component of the evaluation. Potential participants were involved with RTWA from 2008 - 2012. Potential participants were contacted via post and provided with information regarding the evaluation. One 
hundred and forty-three (12.1\%) letters were returned as the participants no longer resided at the postal address. In total, 113 (10.9\%) surveys were received, either completed or partially completed.

\section{Procedure and Measures}

Prior to commencing this component of the evaluation, ethics approval was obtained from the authors' university research and ethics committee. Introductory letters, detailing the nature of the evaluation, were posted to the randomly selected participants two weeks prior to posting the questionnaire booklet. A questionnaire booklet, along with an explanatory statement, was then posted to the participants. The questionnaires included in the booklet were intended to capture a range of information from the clients, including (but not limited to) demographic information, information regarding the RTW process, mental and physical health, consumer satisfaction, and social support. Participants were asked to complete and return the questionnaires in a provided reply paid envelope. Participation was voluntary and former RTWA clients were informed that they would not be penalised if the questionnaires were not returned. Follow-up letters were posted three weeks after the questionnaires had been delivered.

The original Fear-Avoidance Beliefs Questionnaire (FABQ; Waddell et al., 1993) is a self-report measure utilised to assess patient beliefs about the effects of physical activity and work on lower back pain. In the current evaluation, two items, which specifically referenced back injuries, were modified to be non-injury specific (Hart et al., 2009). Including a total of 16 items, the measure is comprised of two subscales, indicating fear of physical activities (FABQ-PA) and the fear of work activities (FABQ-W).

The FABQ has been utilised to assess individuals with different health conditions and has demonstrated good psychometric properties (Crombez, Vlaeyen, Heuts, \& Lysens, 1999; 
Waddell et al., 1993). In the current study, Cronbach’s alpha was .94 for total FABQ, .87 for FABQ-PA, and .88 for FABQ-W. Demonstrating construct validity, the FABQ correlates moderately with the Tampa Scale for Kinesiophobia (Crombez et al., 1999), measuring fear of movement, and has demonstrated good test-retest reliability (Pfingsten et al., 2000; Waddell et al., 1993).

The Short Form-36 Health Survey (Ware, Snow, Kosinski, Gandek, 1993) is an individually administered measure for assessing health-related quality of life (Ware et al., 1993; Ware, Kosinski, Keller, 1994). Consisting of 36 items, the SF-36 yields eight dimensions of functional health and well-being and two summary scores, the Physical Component Summary and the Mental Component Summary, which were utilised in the current study. The Physical Component Summary is comprised of the following subscales: general health, physical functioning, role-physical, and bodily pain, while the Mental Component Summary is comprised of vitality, social functioning, mental health, and roleemotional subscales.

The SF-36 has been used in clinical practice and a variety of research purposes with satisfactory psychometric properties (Ware et al., 1993; Ware et al., 1994). Internal consistency, test-retest reliability, and alternate form reliability (coefficients .68 to .93) for the SF-36 have been found to be acceptable to excellent (Ware et al., 1993; Ware et al., 1994). The SF-36 has demonstrated construct validity in positive correlations with the General Health Rating Index (Read, Quinn, \& Hoefer, 1987), the Quality of Well-Being Scale (Fryback et al., 1993) and the Physical Performance Test (Reuben \& Sui, 1990). Validity studies using the SF-36 support the instrument's ability to differentiate injured and non-injured persons (Ware et al., 1993). 


\section{Results}

Of the 1179 surveys that were posted to potential participants, 113 were returned. Three surveys were excluded from the analysis as these participants reported 0 contacts with RTWA. Six surveys indicating a psychological injury were also excluded from the analysis, given the focus of the FABQ is physical injury. In total 104 surveys were included in the analysis. Some surveys were only partially completed; the number of participants who completed each section is indicated below.

\section{Descriptives}

The sample included 42 males and 40 females, with 22 participants not specifying gender. The average age of participants at time of injury was 46.17 years $(S D=10.97)$ with ages ranging from 21 to 66 years. Participants were asked to report their injury. Utilising the categories from Queensland’s Workers’ Compensation and Rehabilitation Act 2003, these injuries $(n=92)$ were classified as indicated in Table 1 .

Insert table 1 about here

Of the returned surveys where participants reported RTW status $(n=99), 47.8 \%$ reported current employment (RTW; $n=47$ ), 27.3\% reported previous return to work but no current employment (non-durable RTW; $n=27$ ), and 25.3\% reported no return to work (NRTW; $n=25)$. Of those currently working and reporting length of RTW ( $n=44)$, the average length of RTW was 15.19 months $(S D=10.17)$. These values ranged from 2 months to 60 months. Of participants reporting non-durable RTW, 23 participants reported their 
length of time working prior to stopping. The average length of non-durable RTW was 5.00 months $(S D=8.63)$, ranging from less than a month to 42 months.

\section{Analysis of Fear Avoidance Beliefs, Physical Health, and Mental Health}

The SF-36 provides two summary scores, the Physical Component Summary and the Mental Component Summary. Means and standard deviations for the summary scores by RTW status are displayed in Table 2. Higher scores are indicative of higher levels of healthrelated quality of life. Scores ranging from 45 to 55 are considered to be normative with scores below 45 categorised in the clinical range. For all RTW groups, none of the summary scores were normative, demonstrating the prevalence and significant nature of physical and mental health difficulties experienced by the sample.

Insert table 2 about here

An ANOVA indicated significant differences between the RTW groups on the Physical Component Summary, $F(2,94)=6.88, p=.002, \eta^{2}=.13$, with the NRTW group scoring significantly lower than the RTW ( $p=.001)$ and non-durable RTW ( $p=.029)$ groups. No significant differences between the RTW groups were observed for the Mental Component Summary, $F(2,94)=1.40, p=.251, \eta^{2}=.03$.

Previous RTWA clients completed a modified version of the Fear Avoidance Beliefs Questionnaire (FABQ; Waddell et al., 1993). The 16 item FABQ provides a total score in addition to 2 subscales: fear avoidance beliefs regarding physical activity (FABQ-PA) and fear avoidance beliefs regarding work (FABQ-W). Pearson correlation coefficients were utilised to explore the nature of the relationship between fear avoidance beliefs, physical health, and mental health. Displayed in Table 3, fear avoidance beliefs were significantly related to reported physical and mental health. Participants who experienced more health 
related difficulties reported elevated fear avoidance beliefs compared to participants with less health related difficulties.

Insert table 3 about here

ANOVA was utilised to ascertain whether there were significant differences in reported fear avoidance beliefs between the RTW, non-durable RTW, and NRTW groups. Upon histogram inspection, the assumption of normality appeared to be violated for all three of the FABQ scales. Square root transformations of the data were subsequently applied. Because the transformations resulted in improvements to the normality of the distributions, the transformed data was utilised in the following analyses. To aid in the interpretability of the results, the means and standard deviations reported in Table 4 are of the non-transformed data.

Insert table 4 about here

The results of the ANOVA for Total FABQ indicated a significant difference in fear avoidance beliefs according to RTW status, $F(2,80)=4.69, p=.012, \eta^{2}=.10$, with the NRTW group reporting significantly greater fear avoidance beliefs than the RTW group ( $p=$ .008) but not the non-durable RTW group. On the FABQ-Work subscale, no significant differences were noted by RTW status, $F(2,83)=2.62, p=.079, \eta^{2}=.06$. Significant differences between groups were observed on the FABQ-Physical Activity subscale, $F(2,94)$ $=5.71, p=.005, \eta^{2}=.10$. On this subscale, the NRTW group reported significantly greater 
fear avoidance beliefs regarding physical activity than both the RTW group $(p=.020)$ and the non-durable RTW group $(p=.005)$.

An ANCOVA was utilised to ascertain whether the RTW, non-durable RTW, and NRTW groups differed according to fear avoidance beliefs while controlling for overall physical health as measured by the Physical Component score from the SF-36. When controlling for overall physical health, no statistically significant differences on fear avoidance beliefs were observed between the RTW, non-durable RTW, and NRTW groups, $F$ $(2,78)=0.98, p=.38, \eta^{2}=.02$. Of note, the power for detecting a between group difference on fear avoidance beliefs for the ANCOVA was low, .024.

\section{Discussion}

This study examined the relationship between fear avoidance beliefs, health-related quality of life, and their influence on RTW outcomes. Previous research has indicated that fear avoidance beliefs are an important factor in predicting disability, health-related quality of life, and work absence (Fritz et al., 2001; Keely et al., 2008; Waddell et al., 1993). The current study found that, overall, the sample was highly disadvantaged in terms of health, with mean scores of physical and mental health in the clinical range for all RTW outcomes. The durable RTW group reported significantly better physical health than the NRTW group but not the non-durable RTW group. No significant differences were observed between groups on mental health.

The current findings suggest that within the current sample physical health, rather than mental health, was more influential on RTW outcome. This is contrary to previous research suggesting that mental health is a stronger predictor of RTW outcome than physical health (Anderson, Dumont, Azzaria, Le Bourdais, \& Noreau, 2007; Vlasveld et al., 2012). The majority of the current sample have experienced prolonged work absence due to 
occupational injury. Moreover, in order for clients to access RTWA they must be unable to return to their previous employment due to the nature of their injury. While not formally assessed, perhaps overall physical injury severity was higher in the current sample than elsewhere in the literature given these requirements for RTWA program participation. Demonstrating the general severity of injury within the sample, the average reported injury treatment time was 18.77 months $(S D=16.02)$. Another factor that needs to be considered in interpreting these results pertains to the impact of RTW on physical health. It is possible that RTW, and its subsequent physical benefits, may have contributed to the higher levels of physical health reported by the RTW group. This finding also suggests that factors other than physical and mental health contribute to maintaining employment and differentiating the RTW and non-durable RTW groups.

Displayed in Table 3, significant correlations were observed between both work and physical activity related fear avoidance beliefs and measures of physical and mental health. This finding appears consistent with the fear avoidance model proposed by Vlaeyen and Linton (2000). In this model, pain interpreted as threatening results in safety seeking behaviours (e.g., avoidance). These behaviours promote disuse and prevent the disconfirmation of beliefs that normal, functional behaviours are not possible due to the experience of pain, thereby contributing to the maintenance of long-term disability and work absence. These strong correlations may be reflective of the reciprocal relationship between fear avoidance beliefs, physical, and mental health in which fear avoidance beliefs hinder recovery from physical injury, subsequently contributing to a deterioration in mental health. These findings support previous research indicating that the treatment of fear avoidance beliefs may lead to improvements in functional capacity and RTW outcomes (Sullivan et al., 2006). 
Consistent with previous research (Pfingsten et al., 2000), participants with RTW outcomes reported lower fear avoidance beliefs than the NRTW group. While significant group differences were observed on the Total FABQ scale and physical activity related FABQ subscale, group differences on the work related FABQ subscale were approaching significance. However, when controlling for physical health, no fear avoidance group differences were observed. Similar to the findings described above, this may be reflective of the reciprocal relationship between fear avoidance beliefs and physical health in the context of recovery from physical injury. Our findings are inconsistent with Fritz et al. (2001), who found that fear avoidance beliefs were significant predictors of disability and work status, even when controlling for pain intensity and physical impairment. In contrast to the RTWA population where long term work disability was the norm, the Fritz et al. sample had been work disabled for four weeks at the time of assessment. The findings for the current study also contrast Waddell et al. (1993) who found that fear avoidance beliefs accounted for significant variance of work disability, after controlling for severity of pain (bodily pain is one of the subscales in the Physical Health Composite of the SF36). The sample utilised in Waddell et al. was more comparable to the RTWA population with mean length of work disability due to low back pain of approximately four months. It is quite likely the failure to detect a significant between group difference on fear avoidance, whilst controlling for physical health, reflects the low power obtained for the current analyses. Replication and extension with a larger sample size is indicated to further evaluate the association between fear avoidance and RTW outcomes.

Clients with poorer physical health are likely to require more intensive support in managing both their injury related physical issues and injury related beliefs if a successful RTW outcome is to be achieved. These findings from the current study and previous research suggest that for clients with prolonged work absence, collaborative practice between medical practitioners, psychologists, and physiotherapists is likely to be required to improve 
RTW outcomes for this complex client group. Such collaboration should include the development of a RTW plan and regular communication between the parties involved, including the client. Treatment strategies should include screening participants for psychological, as well as physical difficulties, at the commencement of treatment. As fear avoidance beliefs have been observed as early as five days post-injury (Coudeyre et al., 2007), identifying and addressing such beliefs early in the treatment process may decrease the likelihood of long-term disability and work absence. The identification of individuals at risk of long-term work absence may also allow for the most effective allocation of resources.

Cognitive behavioural therapy is based on the premise that underlying beliefs influence behaviour. Challenging and modifying fear avoidance beliefs is likely to result in more adaptive behaviours conducive to effective rehabilitation and return to work. A cognitive behavioural approach to treatment including graded exposure to physical activity has been advocated as an effective treatment strategy (Crombez et al, 1999). Behavioural experiments allow the explicit testing of fear avoidance cognitions and beliefs. Research has supported such an approach with decreased pain related fear through cognitive behavioural therapy demonstrated to increase functional capacity and positive RTW outcomes (Vlaeyen, de Jong, Geilen, Heuts, \& van Breukelen, 2002).

The low response rate was a limitation of the current study. Of the 1179 questionnaires posted to participants, 113 (10.9\%) were returned. This low response rate may have resulted in a sampling bias, decreasing the representativeness of the sample. The current study's correlational design limited the inferences that could be drawn regarding causality, particularly the relationship between physical health and fear avoidance beliefs. Future studies employing a prospective or experimental design are required to further elicit the nature of this relationship. In interpreting the study findings it is also important to note, 
that while the average length of RTW for the RTW group was nearly 15 months, some of the participants in this group may still stop working.

This study was unique in that it compared fear avoidance beliefs and health-related quality of life according to RTW status: RTW, non-durable RTW, and NRTW. Collaborative practice amongst professionals, particularly medical practitioners, psychologists, and physiotherapists, that addresses both fear avoidance beliefs and physical health is likely to promote patient recovery from injury and decrease disability, including work absence. 


\section{Acknowledgement}

The authors would like to thank the Return to Work Assist and Data Management and Analytics personnel with Q-Comp for the assistance in establishing the research.

\section{Declaration of Interest}

The project was funded by Q-Comp, an independent statutory authority, established on 1 July 2003 under the Workers' Compensation and Rehabilitation Act 2003 to oversee Queensland's workers' compensation scheme. 


\section{References}

Anderson, D., Dumont, S., Azzaria, L., Le Bourdais, M., \& Noreau, L. (2007) Determinants of return to work among spinal cord injury patients: A literature review. Journal of Vocational Rehabilitation, 27, 57-68.

Burton, A., Tillotson, K., \& Main, C. (1995). Psychosocial predictors of work loss in acute and subchronic low back trouble. Spine, 20, 722-728. 1995; 20: 722-728.

Coudeyre, E., Tubach, F., Rannou, F., Baron, G., Coriat, F., Brin, S.,...Poiraudeau, S. (2007). Fear-avoidance beliefs about back pain in patients with acute low back pain. Clinical Journal of Pain, 23, 720-725.

Crombez, G., Vlaeyen, J., Heuts, P., \& Lysens, R. (1999). Pain-related fear is more disabling than fear itself: Evidence on the role of pain-related fear in chronic back pain disability. Pain, 80, 329-339.

Dekkers-Sanchez, P., Wind, H., Sluiter, J., \& Frings-Dresen, M. (2010). Qualitative study of perpetuating factors for long term sick leave and promoting factors for return to work: Chronic work disabled patients in their own words. Journal of Rehabilitation Medicine, 42, 544-552.

Fryback, D., Dasbach, E., Klein, R., Klein, B., Dorn, N., Peterson, K., \& Martin, P. (1993). The Beaver Dam Outcomes Study: Initial catalog of health-state quality factors. Medical Decision Making, 13, 89-102. 
Hart, D., Werneke, M., George, S., Matheson, J., Wang, Y., Cook, K.,...Choi, S. (2009). Screening for elevated levels of fear-avoidance beliefs regarding work or physical activities in people receiving outpatient therapy. Physical Therapy, 89, 770-785.

Fritz, J., George, S., Delitto, A. (2001). The role of fear-avoidance beliefs in acute low back pain: Relationships with current and future disability and work status. Pain, 94, 7-15.

Keely, P., Creed, F., Tomenson, B., Todd, C., Borglin, G., \& Dickens, C. (2008). Psychosocial predictors of health-related quality of life and health service utilisation in people with chronic low back pain. Pain, 135, 142-150.

Macfarlane, G., Thomas, E., Croft, P., Papageorgiou, A., Jayson, M., \& Silman, A. (1999). Predictors of early improvement in low back pain amongst consulters to general practice: The influence of pre-morbid and episode-related factors. Pain, 80, 113-119.

Pfingsten, M., Kroner-Herwig, B., Leibing, E., Kronshage, U., \& Hildebrand, J. (2000). Validation of the German version of the fear-avoidance belief questionnaire (FABQ). European Journal of Pain, 4, 259-266.

Read, J., Quinn, R., \& Hoefer, M. (1987). Measuring overall health: an evaluation of three important approaches. Journal of Chronic Diseases, 40 (Suppl. 1), 7S-26S.

Reuben, D. \& Sui, A. (1990) An objective measure of physical function of elderly outpatients: The physical performance test. Journal of the American Geriatrics Society, 38, 1105-1112. 
Rose, M., Klenerman, L., Atchison, L., \& Slade, P. (1992). An application of the fear avoidance model to three chronic pain problems. Behaviour Research and Therapy, 30, 359-365.

Safework Australia (2012). Australian work health and safety strategy 2012-2022. Canberra: Author. http://www.safeworkaustralia.gov.au/sites/SWA/about/Publications/Documents/719/ Australian-WHS-Strategy-2012-2022.pdf. Accessed 15 June 2013.

Sullivan, M., Adams, H., Thibault, P., Corbiere, M., \& Stanish, W. (2006). Initial depression severity and the trajectory of recovery following cognitive-behavioural intervention for work disability. Journal of Occupational Rehabilitation, 16, 63-74.

Szymanski, E., \& Hershenson, D. (1998). Career Development of people with disabilities: An ecological model. In R. Parker \& E. Szymanski (Eds.). Career Choice and Development ( $3^{\text {rd }}$ ed.) (pp. 327-378). Austin, TX: Pro-ed.

Turk, D. (2002). A diathesis-stress model of chronic pain and disability following traumatic injury. Pain Research and Management, 7, 9-20.

Vlasveld, M., van der Feltz-Cornelis, C., Bultmann, U., Beekman, A., van Mechelen, W., Hoedeman, R., \& Anema, J. (2012). Predicting return to work in workers with allcause sickness absence greater than 4 weeks: a prospective cohort study. Journal of Occupational Rehabilitation, 22, 118-126.

Vlayen, J. \& Linton S. (2000). Fear-avoidance and its consequences in chronic musculoskeletal pain: A state of the art. Pain, 85, 317-332. 
Vlaeyen, J., de Jong, J., Geilen, M., Heuts, P., \& van Breukelen, G (2002). The treatment of fear movement/(re)injury in chronic low back pain: further evidence on the effectiveness of exposure in vivo. Clinical Journal of Pain, 18, 251-261.

Waddell, G., Burton, K., \& Kendall, N. (2008). Vocational rehabilitation: What works, for whom, and when. Vocational Rehabilitation Task Group. https://www.gov.uk/government/uploads/system/uploads/attachment_data/file/209474 /hwwb-vocational-rehabilitation.pdf. Accessed 17 August 2013.

Waddell, G., Newton, M., Henderson, I., Somerville, D., \& Main, C. (1993). A Fear Avoidance Beliefs Questionnaire (FABQ) and the role of fear-avoidance beliefs in chronic low back pain and disability. Pain, 52, 157-168.

Ware, J., Snow, K., Kosinski, M., \& Gandek, B. (1993). SF-36 Health Survey manual and interpretation guide. Boston: Health Institute.

Ware, J., Kosinski, M., \& Keller, S. (1994). SF-36 physical and mental health summary scales. Boston: Health Institute. 
Table 1

Nature of the Reported Injuries within the Sample

Type of Injury $\quad n \quad \%$

Upper Extremity 29

31.5

Lower Extremity

20

21.7

Musculoskeletal

27

29.3

Multiple Injuries

16

17.4 
Table 2

SF-36 Physical and Mental Component Means and Standard Deviations

$\begin{array}{lll}\text { SF-36 Scale } & M & S D\end{array}$

Physical Component Summary

RTW

Non-durable RTW

NRTW

Mental Component Summary

RTW

42.05

13.68

Non-durable RTW

37.21

15.07

NRTW

37.52

12.58

Note. RTW $=$ Return to Work; NRTW $=$ No Return to Work

${ }^{*} p<.01 ; * * p<.05$ 
Table 3

Correlations of Fear Avoidance Beliefs, Physical Health, and Mental Health

\begin{tabular}{|c|c|c|c|c|}
\hline Variable & 1 & 2 & 3 & 4 \\
\hline 1. FABQ-W & - & $.71 *$ & $.57 *$ & $.50 *$ \\
\hline 2. FABQ-PA & & - & $.61 *$ & $.40 *$ \\
\hline 3. Physical Component & & & - & $.38 *$ \\
\hline 4. Mental Component & & & & - \\
\hline
\end{tabular}

Note. FABQ-W = Fear Avoidance Beliefs Questionnaire - Work; FABQ-PA = Fear Avoidance Beliefs Questionnaire - Physical Activity

$* p<.001$ 
Table 4

Means and Standard Deviations of Fear Avoidance Beliefs by RTW Status

$\begin{array}{lll}\text { FABQ Scale } & M & S D\end{array}$

Total FABQ

$\operatorname{RTW}(n=40)$

60.55

22.62

Non-durable RTW $(n=23)$

65.74

26.75

$\operatorname{NRTW}(n=20)$

$77.00 *$

FABQ-Work

RTW

27.02

10.81

Non-durable RTW

30.00

11.61

NRTW

32.52

12.48

FABQ-Physical Activity

RTW

$15.95 * *$

6.01

Non-durable RTW

$14.67 *$

6.92

NRTW

19.40

6.53

Note. FABQ = Fear Avoidance Beliefs Questionnaire; RTW = Return to Work; NRTW = No Return to Work

${ }^{*} p<.01 ;{ }^{* *} p<.05$ 